\title{
Diagnosis of Hurler's Syndrome in the Hospital Laboratory and the Determination of its Genetic Type
}

\author{
G. MANLEY and J. HAWKSWORTH \\ From the Nuffield Department of Clinical Biochemistry, Radcliffe Infirmary, Oxford
}

The rare genetically determined disease now known as Hurler's syndrome, characterized by dwarfism, grotesque skeletal deformity, limitation of joint movement, deafness, hepatosplenomegaly, cardiac abnormality, characteristic facies, and perhaps clouding of the cornea and mental retardation (McKusick, 1960), was first described by Hunter (1917). For some years, the substance that accumulates in the tissues in this condition was thought to be a lipid-largely because it disappeared during routine histological processing-and this gave rise to the term lipochondrodystrophy. However, the work of Dorfman and Lorincz (1957) and Meyer, Grumbach, Linker, and Hoffman (1958) showed that mucopolysaccharides were excreted in excess in the urine, and accumulated in the tissues. More recently, Terry and Linker (1964) have shown that the different genetic forms of Hurler's syndrome excrete different patterns of mucopolysaccharide in the urine.

Several laboratory tests have been devised to aid the diagnosis of Hurler's syndrome, though none has received universal acceptance. A turbidity reaction between mucopolysaccharides and acidified albumin, developed by Dorfman and Ott (1948) for the assay of hyaluronidase, has been adapted to the examination of urine (McKusick, 1960). Berry and Spinanger (1960) described a paper spot test for Hurler's syndrome, utilizing the metachromatic reaction between sulphated mucopolysaccharides and toluidine blue. Segni, Romano, and Tortorolo (1964) found that the uronic acid content of dialysed urine was higher in cases of Hurler's syndrome than in controls, and suggested this as a diagnostic test. For the haematologist, metachromatic inclusions in polymorphs (Reilly, 1941) and lymphocytes (Muir, Mittwoch, and Bitter, 1963) afford a diagnosis in many cases.

Though a combination of these investigations may

Received July 14, 1965. provide a reliable laboratory diagnosis of Hurler's syndrome, isolation and fractionation of urinary mucopolysaccharides by conventional methods, such as stepwise saline elutions from ion exchange columns (Schiller, Slover, and Dorfman, 1961) and their full characterization by glucosamine, galactosamine, glucuronic acid, iduronic acid, and sulphate assays, are beyond the scope of most hospital laboratories. This paper describes a simple urine screening test for Hurler's syndrome, and a method of determining the urinary mucopolysaccharide pattern, that can be carried out in the hospital biochemistry laboratory.

\section{Methods}

Urine samples from 100 random controls and 7 cases of Hurler's syndrome were examined. Full 24-hour collection of urine was found to be essential as mucopolysaccharide excretion varied throughout the day. The urine samples were not adjusted to a fixed volume or creatinine content as this was found to be unnecessary and in any case unreliable when applied to children of widely differing ages. Merthiolate was a satisfactory preservative.

Screening: the CPC turbidity test. This test was based on the formation of an insoluble complex between mucopolysaccharides (MPS) and cetylpyridinium chloride (CPC). This reaction has been used for the isolation of mucopolysaccharides from tissues (Scott, 1960). An aliquot of urine $(20 \mathrm{ml}$.) from the 24-hour sample was centrifuged, and the clear supernatant $(5 \mathrm{ml}$.) was pipetted into each of two tubes, 'test' and 'blank'. Cetylpyridinium chloride (Koch-Light Laboratories, Colnbrook, Bucks.) $0 \cdot 1 \% \mathrm{w} / \mathrm{v}$ in water $(5 \mathrm{ml}$.) was added to 'test', and mixed by inversion. Water ( $5 \mathrm{ml}$.) was added to 'blank', and mixed. The optical density (OD) of 'test' was read against 'blank' 4 min. after adding CPC in a Unicam S.P. 300 with $1 \mathrm{~cm}$. cells and Ilford 625 filter. $C P C$ turbidity = OD 'test' vs. 'blank' 4 min. after adding CPC. The time interval was important as maximal OD was reached after $4 \mathrm{~min}$. and thereafter fell due to aggregation of particles. 


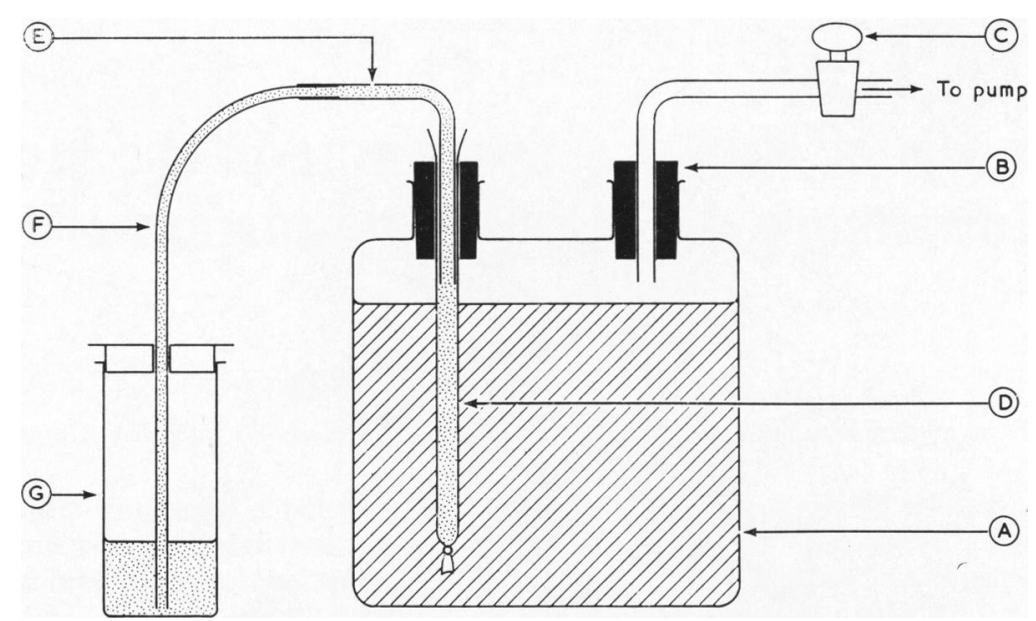

FIG. 1.-Apparatus for concentrating urine. For explanation, see text.

Uronic acid content of CPC precipitate. All abnormal CPC turbidity readings were further investigated by measuring the uronic acid content of the mucopolysaccharides precipitated by CPC. In order to do this, it was necessary to dissociate the MPS-CPC complex and isolate the MPS. After reading OD in the CPC turbidity test, the whole of the test solution was centrifuged, and the deposit washed with 2 changes $(1 \mathrm{ml}$. each) of $0.1 \%$ CPC. The complex was then dissolved in $2 \mathrm{M} . \mathrm{NaCl}(1 \mathrm{ml}$.) and, after centrifuging, the clear supernatant was transferred to $95 \%$ ethanol (5 ml.). The precipitated MPS was isolated by centrifugation, washed with $95 \%$ ethanol $(1 \mathrm{ml}$.$) , dried, and taken up in$ water $(1 \mathrm{ml}$.). Uronic acid was assayed by the method of Bitter and Muir (1962) on $1: 1$ and $1: 10$ dilutions of this MPS solution, and results expressed as mg. uronic acid/1. urine.

Determination of urinary mucopolysaccharide pattern. A macromolecular concentrate of urine was obtained by ultrafiltration under a vacuum combined with dialysis against water, and the mucopolysaccharides in this concentrate were separated by electrophoresis on cellulose acetate membrane.

Vacuum dialysis of urine. The apparatus originally used by Prof. G. Hunter for concentrating CSF protein is shown in Fig. 1. A Woulff's bottle (A) of $500 \mathrm{ml}$. capacity containing $400 \mathrm{ml}$. water was fitted with two single-hole rubber bungs (B). A glass tube with a rightangle bend and a tap (C) was fitted into one bung and connected to a water-suction pump. A length ( 9 in. $(23 \mathrm{~cm})$.$) of 8 / 32$ in. $(0 \cdot 64 \mathrm{~cm}$.) Visking dialysis tubing (D) (Hudes Merchandising Co., London) was cut and knotted securely at one end. The open end was then passed upwards through the remaining bung, and a short length of glass tubing with a right angle bend and rounded ends was passed through the bung, inside the viscose tubing. Tight fit was essential. A length of polythene catheter $(F)$ that fitted tightly inside the glass tubing (E) was passed through a hole in the lid of a polythene container ( $G$ ) to touch the bottom. The urine sample $(15 \mathrm{ml}$.) was centrifuged, and the clear supernatant $(10 \mathrm{ml}$.) transferred to the polythene container. The water pump was turned on, and the tap (C) opened. The urine was thus aspirated into the dialysing sac, and allowed to concentrate overnight $(15 \mathrm{hr}$.). The vacuum was then released, and the small residue remaining in the sac was subjected to electrophoresis.

Electrophoresis of urine concentrates on cellulose acetate. Samples of the residue $(10 \mu \mathrm{l}$.) were applied to cellulose acetate strips $(20 \times 5 \mathrm{~cm}$., Oxoid) that had been allowed to equilibrate for 1 hour in an electrophoresis tank containing Michaelis' sodium barbiturate-acetate buffer $p \mathrm{H} 9 \cdot 2$. Standard samples of hyaluronic acid, heparitin sulphate, and chondroitin sulphates $B$ and $C$, prepared from human umbilical cord and human aorta by papain digestion, CPC precipitation, and Dowex-1 column chromatography (Manley, 1965) were run alongside urine samples. Electrophoresis was carried out for 80 minutes with a constant potential gradient of $20 \mathrm{~V} / \mathrm{cm}$. at $20^{\circ} \mathrm{C}$. The strips were then transferred without drying to $1 \% \mathrm{w} / \mathrm{v}$ Alcian Blue (G.T. Gurr) in $2 \% \mathrm{v} / \mathrm{v}$ acetic acid (30 min.) and washed in running tap water $(30 \mathrm{~min}$.). They were dried between sheets of Whatman No. 1 filter paper. Duplicate strips were stained in $0.2 \% \mathrm{w} / \mathrm{v}$ Light Green in 3\% v/v trichloracetic acid, and in $0.1 \% \mathrm{w} / \mathrm{v}$ Azure $\mathrm{A}$ in $70 \%$ ethanol.

Scanning of strips. After clearing in liquid paraffin, the strips were scanned in a Chromoscan (Joyce-Loeble $\&$ Co.), using filter 607 and slit 5006. The count from each Alcian Blue-positive band was expressed as a percentage of the total count. 


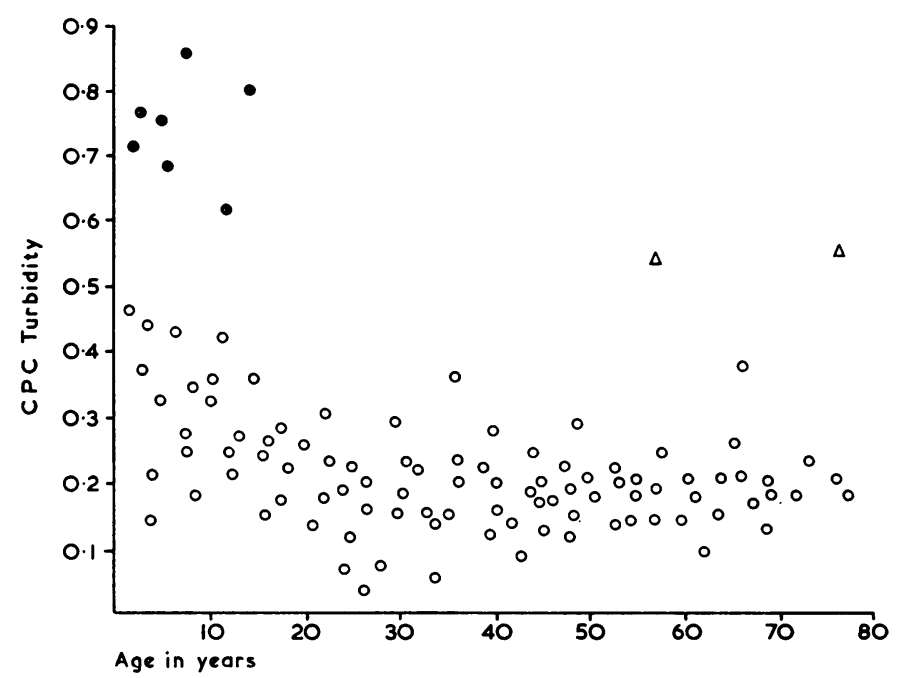

FIG. 2.-CPC turbidity of urines from 98 controls $\mathrm{O}$, and 7 cases of Hurler's syndrome $\mathrm{O}$, plotted against age. Triangles $\triangle$ represent 2 controls with high urine muco-protein content.

\section{Results}

The CPC turbidity of urine from 100 random control cases, compared with 7 cases of Hurler's syndrome, is shown in Fig. 2. $98 \%$ of the control cases gave readings within the range $0 \cdot 1-0.5$ with a tendency towards the upper limit in early childhood. The urine from cases of Hurler's syndrome gave consistently high turbidity readings varying from 0.62 to 0.85 .

The urine uronic acid content, as determined on the mucopolysaccharides isolated from the CPC precipitate, was low (1-5 mg./1.) in control cases and high (20-60 mg./1.) in cases of Hurler's syndrome. The 2 control cases with a CPC turbidity above 0.5 gave a uronic acid level well within the normal range.

Electrophoresis of urine concentrates produced in every case several Alcian Blue-positive bands that were identified as follows. A rapidly migrating Alcian Blue-positive band corresponding in mobility with the chondroitin sulphates, showing a metachromatic reaction with Azur-A, and failing to stain with Light Green, was designated chondroitin sulphate. 2 (sometimes 1 or 3 ) slowly migrating bands, which failed to give a metachromatic reaction with Azure-A but stained intensely with Light Green, were designated 'miscellaneous mucoprotein'. A band of intermediate mobility corresponding with heparitin sulphate, which gave a metachromatic reaction with Azur A and failed to stain with Light Green, was designated heparitin sulphate. A compact band corresponding in mobility and staining reactions with hyaluronic acid was found infrequently.
Chondroitin sulphate, heparitin sulphate, and mucoprotein were found in all urines examined. Chondroitin sulphate was the major urinary mucopolysaccharide in early childhood, but chondroitin sulphate and heparitin sulphate were present in roughly equal proportions in most adults. The mucoprotein bands were very variable, but formed an exceedingly high proportion of the Alcian Bluepositive material in the 2 control cases that gave a high turbidity reading (Fig. 4).

The cases of Hurler's syndrome were easily recognized on electrophoresis, because of the great excess of Alcian Blue-positive material, and two quite different patterns were found. In 4 cases, both chondroitin sulphate and heparitin sulphate were present in large quantities and roughly equal proportions. In each of these cases, the heparitin sulphate band could be separated by careful electrophoresis into 2 distinct fractions (Fig. 3b). In the 2 remaining cases, which tended to be the more severely affected clinically, chondroitin sulphate alone was present in great excess (Fig. 3a). Indeed, in both these cases the urine concentrate had to be diluted $1: 5$ with water before satisfactory staining of the chondroitin sulphate band could be obtained.

Chromoscan tracings of electrophoretic strips obtained from the two forms of Hurler's syndrome, from a normal control, and from a control giving a false positive CPC turbidity test due to excess urinary mucoprotein are shown in Fig. 4 for comparison. The relative proportions of the three 


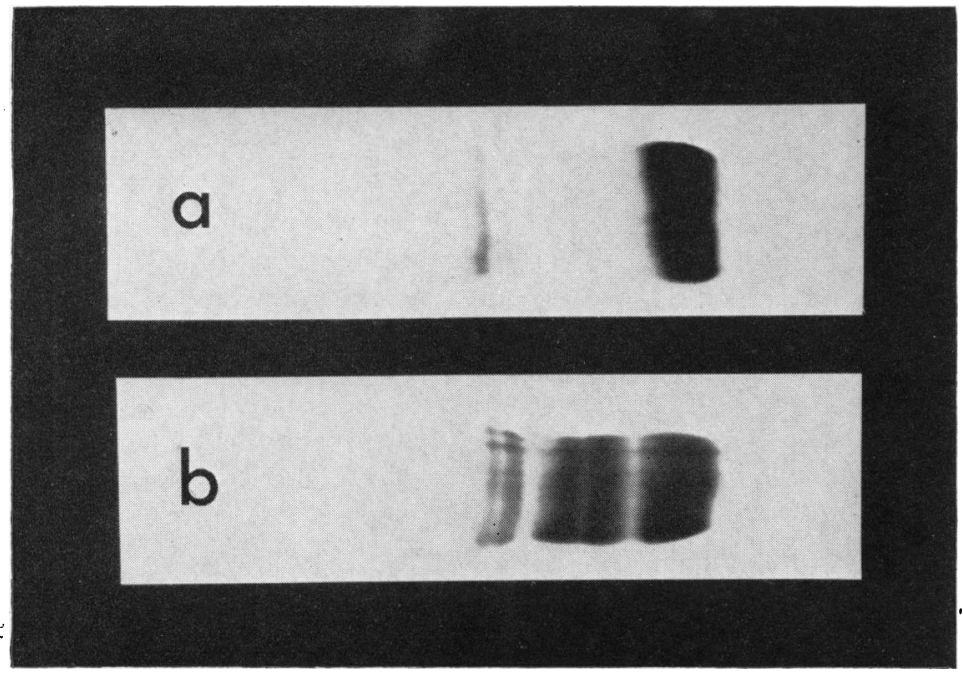

FIG. 3.-Electrophoretic strips of urinary mucopolysaccharide stained with Alcian Blue, from (a) a case of Hurler's syndrome showing excessive excretion of chondroitin sulphate, and (b) a case of Hurler's syndrome showing excessive excretion of both heparitin sulphate and chondroitin sulphate. It can be seen that the 'heparitin sulphate' consists of two distinct fractions. Direction of electrophoretic migration from left to right. For identification of bands, see text.

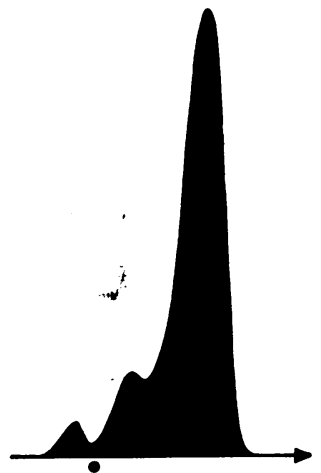

(a)

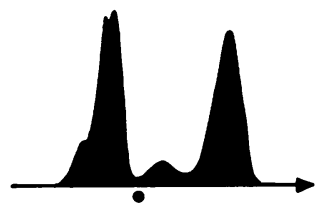

(c)

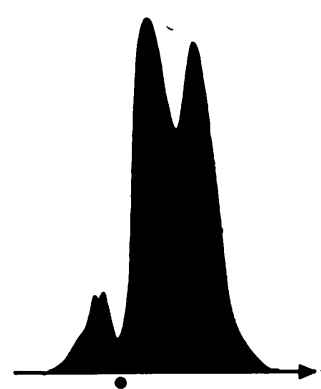

(b)

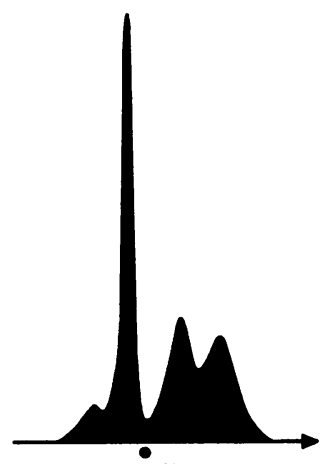

(d)
TABLE I

Relative Proportions of Miscellaneous Mucoprotein Heparitin Sulphate, and Chondroitin Sulphate, Expressed as Percentage of total Mucopolysaccharide in Urine of 7 cases of Hurler's Syndrome

\begin{tabular}{|c|c|c|c|c|c|}
\hline \multirow[b]{2}{*}{ Case } & \multirow[b]{2}{*}{ Sex } & \multirow[b]{2}{*}{$\begin{array}{l}\text { Age } \\
\text { (yr.) }\end{array}$} & \multicolumn{3}{|c|}{$\%$ Total Mucopolysaccharides } \\
\hline & & & $\begin{array}{c}\text { Miscella- } \\
\text { neous } \\
\text { Muco- } \\
\text { protein }\end{array}$ & $\begin{array}{l}\text { Heparitin } \\
\text { Sulphate }\end{array}$ & $\begin{array}{l}\text { Chondro- } \\
\text { itin } \\
\text { Sulphate }\end{array}$ \\
\hline $\begin{array}{c}\text { DM } \\
\text { CM } \\
\text { PS } \\
\text { SN } \\
\text { SB } \\
\text { AFI } \\
\text { AFO }\end{array}$ & $\begin{array}{l}\mathbf{M} \\
\mathbf{M} \\
\mathbf{M} \\
\mathbf{M} \\
\mathbf{M} \\
\mathbf{M} \\
\mathbf{M}\end{array}$ & $\begin{array}{c}13 \\
11 \\
8 \\
43 \\
5 \\
13 \\
3\end{array}$ & $\begin{array}{r}11 \cdot 9 \\
11 \cdot 7 \\
9.6 \\
5 \cdot 6 \\
7.5 \\
3 \cdot 8 \\
3 \cdot 2\end{array}$ & $\begin{array}{r}40 \cdot 6 \\
47 \cdot 6 \\
50 \cdot 5 \\
50 \cdot 8 \\
11 \cdot 8 \\
8 \cdot 7 \\
12 \cdot 6\end{array}$ & $\begin{array}{l}47 \cdot 5 \\
40 \cdot 7 \\
39 \cdot 9 \\
43 \cdot 6 \\
80 \cdot 7 \\
87 \cdot 5 \\
84 \cdot 2\end{array}$ \\
\hline
\end{tabular}

FIG. 4.-Densitometer (Chromoscan) records of urinary mucopolysaccharides, separated by electrophoresis on cellulose acetate and stained with Alcian Blue, from (a) a case of Hurler's syndrome excreting chondroitin sulphate only in excess, (b) a case of Hurler's syndrome excreting both heparitin sulphate and chondroitin sulphate in excess, (c) a normal child, and (d) a case of urinary infection giving a false positive CPC turbidity because of high urinary mucoprotein content. Direction of electrophoretic migration from left to right, as indicated by the arrows. The small black spots immediately below the baseline indicate the position of marker samples of hyaluronic acid. 
major Alcian Blue-positive bands in each case of Hurler's syndrome are shown in the Table.

\section{Discussion}

The CPC turbidity test is very simple to perform, and taking an upper limit of 0.5 for control cases, no false negative results have yet been encountered in proven cases of Hurler's syndrome. However, the rarity of the disease has made it impossible to scan a large series, and until this has been done the possibility of a false negative result cannot be excluded.

In the absence of genito-urinary disease, false positive results seem to be unlikely, but two possible sources of error may be the Morquio-Brailsford syndrome and Marfan's syndrome, in both of which increased mucopolysaccharide excretion has been reported (Robins, Stevens, and Linker, 1963; Berenson and Serra, 1959). Abnormal mucopolysaccharide excretion has also been noted in rheumatoid arthritis (Di Ferrante, 1957), but we have obtained normal CPC turbidity readings in this condition. In the presence of genito-urinary disease (cystitis, pyelonephritis) two false positive results were encountered with the CPC turbidity test, but in both these cases the uronic acid content of the CPC precipitate was well within normal limits, and electrophoresis showed that the positive turbidity test was due to an excess of mucoprotein (Fig. 4d). The presence of protein or Bence-Jones protein in the urine did not influence the CPC turbidity. Technically, there is little to go wrong with the turbidity test, though a false negative may result if the time interval of 4 minutes is ignored.

The estimation of uronic acid in the CPC precipitate is somewhat time consuming and is considered unnecessary if electrophoresis of the urine concentrate is to be carried out. The uronic acid content of the CPC precipitate as estimated here does not give an accurate measure of the total mucopolysaccharide-uronic acid in the urine, as more elaborate techniques (Muir et al., 1963) yield higher results.

Cellulose acetate electrophoresis of the urine concentrate provides a considerable amount of information and is not difficult to perform. In control cases, the volume of urine concentrate is small (usually 10-20 $\mu \mathrm{l}$.) and the entire contents of the dialysis sac may be applied to the strips. In some cases of Hurler's syndrome (particularly the autosomal recessive form) the urine concentrate is larger (approx. $100 \mu \mathrm{l}$.) and needs diluting before electrophoresis, because if too much mucopolysaccharide is present, the edges of the bands will stain brilliantly with Alcian Blue while the central portions will remain unstained. Large amounts of protein interfere with both concentration and electrophoresis, so if heavy proteinuria is present it is advisable to remove protein by heat denaturation before concentration.

Terry and Linker (1964) showed that the autosomal recessive forms of Hurler's syndrome excrete predominantly chondroitin sulphate B in the urine, and the sex-linked recessive forms excrete heparitin sulphate and chondroitin sulphate $B$ in roughly equal proportions. The electrophoretic pattern of the urine concentrates differentiates clearly between these two major forms of Hurler's syndrome. We have not yet encountered the atypical form, described by Terry and Linker, which excretes only heparitin sulphate in excess.

The figures obtained with the Chromoscan for the percentage distribution of mucopolysaccharides on Alcian Blue-stained cellulose acetate electrophoretic strips agree very closely with figures obtained by $\mathrm{NaCl}$-gradient elution of the mucopolysaccharide mixtures from Dowex 1-Cl columns, with subsequent uronic acid assay of column effluent fractions. Indeed the separation achieved by cellulose acetate electrophoresis tends to be sharper than that obtained by column chromatography, and it is interesting that we have been able to separate the 'heparitin sulphate' excreted by the sex-linked form of Hurler's syndrome into two distinct fractions, both of which are resistant to testicular hyaluronidase. The inference is that two types of 'heparitin sulphate' are present, and if they can be separated by column chromatography it may be possible to elicit a difference in molecular structure, perhaps in degree or position of sulphation, perhaps in the configuration of the uronic acid.

The basic defect in Hurler's syndrome remains obscure, and awaits further elucidation of the biosynthesis, tissue fixation, and degradation of mucopolysaccharides. However, the turbidity test described here affords a simple means of screening urine for excessive mucopolysaccharide content, and the electrophoresis of urine concentrates on cellulose acetate membrane offers a method of determining the urinary mucopolysaccharide pattern, and hence the genetic type, of cases of Hurler's syndrome, and can be carried out in most hospital biochemistry laboratories.

\section{Summary}

A simple method of screening urine for cases of Hurler's syndrome, based on a precipitation reaction between the polycationic detergent cetyl pyridinium chloride and polyanionic mucopolysaccharides, is described. Concentration of the 
macromolecular components of urine by ultrafiltration, followed by electrophoresis on cellulose acetate and staining with Alcian Blue, provides excellent separation of the urinary mucopolysaccharides, detects false positive precipitation reactions due to excess mucoprotein, and distinguishes clearly between the automsomal recessive form of Hurler's syndrome, which excretes chondroitin sulphate in excess, and the sex-linked recessive form, which excretes both heparitin sulphate and chondroitin sulphate in excess.

We would like to thank Mr. J. R. P. O'Brien for facilities and helpful criticism, Dr. A. H. T. Robb-Smith for many useful suggestions, and Drs. F. S. W. Brimblecombe, B. Kirman, I. S. Menzies, J. Stern, and Prof. Sir Alan Moncrieff for allowing us to study urine from their cases of Hurler's syndrome.

\section{REFERENCES}

Berenson, G. S., and Serra, M. T. (1959). Mucopolysaccharides in urine from patients with Marfan's syndrome. Fed. Proc., 18, 190.

Berry, H. K., and Spinanger, J. (1960). A paper spot test useful in study of Hurler's syndrome. $\quad \mathcal{F}$. Lab. clin. Med., 55, 136.

Bitter, T., and Muir, H. M. (1962). A modified uronic acid carbazole reaction. Analyt. Biochem., 4, 330.
Di Ferrante, N. (1957). Urinary excretion of acid mucopolysaccharides by patients with rheumatoid arthritis. Fed. Proc., 16, 172.

Dorfman, A., and Lorincz, A. E. (1957). Occurrence of urinary acid mucopolysaccharides in the Hurler syndrome. Proc. nat. Acad. Sci. (Wash.), 43, 443.

- and Ott, M. L. (1948). A turbidimetric method for the assay of hyaluronidase. F. biol. Chem., 172, 367.

Hunter, C. (1917). A rare disease in two brothers. Proc. roy. Soc. Med., 10, (Sect. for Study Dis. Child.), 104.

Manley, G. (1965). Changes in vascular mucopolysaccharides with age and blood pressure. Brit. F. exp. Path., 46, 125.

McKusick, V. A. (1960). Heritable Disorders of Connective Tissue, 2nd ed., pp. 244 and 276. C. V. Mosby Co., St. Louis.

Meyer, K., Grumbach, M. M., Linker, A., and Hoffman, P. (1958). Excretion of sulfated mucopolysaccharides in gargoylism (Hurler's syndrome). Proc. Soc. exp. Biol. (N.Y.), 97, 275.

Muir, H., Mittwoch, U., and Bitter, T. (1963). The diagnostic value of isolated urinary mucopolysaccharides and of lymphocyte inclusions in gargoylism. Arch. Dis. Childh., 38, 358.

Reilly, W. A. (1941). The granules in the leukocytes in gargoylism. Amer. F. Dis. Child., 62, 489.

Robins, M. M., Stevens, H. F., and Linker, A. (1963). Morquio's disease: an abnormality of mucopolysaccharide metabolism. f. Pediat., 62, 881 .

Schiller, S., Slover, G. A., and Dorfman, A. (1961). A method for the separation of acid mucopolysaccharides: its application to the isolation of heparin from the skin of rats. $\mathcal{f}$. biol. Chem., 236, 983.

Scott, J. E. (1960). Aliphatic ammonium salts in the assay of acidic polysaccharides from tissues. Meth. biochem. Anal., 8, 145.

Segni, G., Romano, C., and Tortorolo, G. (1964). Diagnostic test for gargoylism. Lancet, 2, 420.

Terry, K., and Linker, A. (1964). Distinction among four forms of Hurler's syndrome. Proc. Soc. exp. Biol. (N.Y.), 115, 394. 